Pacific

Journal of

Mathematics

\title{
AREA, WIDTH, AND LOGARITHMIC CAPACITY OF CONVEX SETS
}

Roger W. Barnard, Kent Pearce, and Alexander Yu. Solynin 


\section{AREA, WIDTH, AND LOGARITHMIC CAPACITY OF CONVEX SETS}

Roger W. Barnard, Kent Pearce, and Alexander Yu. Solynin

For a planar convex compact set $E$, we describe the mutual range of its area, width, and logarithmic capacity. This result will follow from a more general theorem describing the mutual range of area, logarithmic capacity, and length of orthogonal projection onto a given axis of an arbitrary compact set, connected or not.

\section{Introduction.}

For a planar convex compact set $E$, let $A(E), w(E)$, and cap $E$ denote the area, width, and logarithmic capacity of $E$ respectively. The width $w(E)$ is the minimal orthogonal projection of $E$, i.e.,

$$
w(E)=\min _{0 \leq \theta \leq \pi} \operatorname{proj}_{\theta} E,
$$

where $\operatorname{proj}_{\theta} E$ denotes the length of the orthogonal projection of $E$ onto the line $l_{\theta}=\left\{z=t e^{i \theta}:-\infty<t<\infty\right\}$. The logarithmic capacity cap $E$ of a compact set $E$ is defined by

$$
-\log \operatorname{cap} E=\lim _{z \rightarrow \infty}(g(z)-\log |z|),
$$

where $g(z)$ denotes Green's function of the unbounded component $\Omega(E)$ of $\overline{\mathbb{C}} \backslash E$ having singularity at $z=\infty$. This notion combines several characteristics of a compact set such as transfinite diameter, Chebyshev's constant, and outer radius, see $[\mathbf{3}, \mathbf{4}, \mathbf{7}, \mathbf{8}, \mathbf{1 0}]$ and $[\mathbf{1 2}]$.

How large can the area of $E$ be if the width and logarithmic capacity of $E$ are prescribed? - For convex sets, the answer to this question is given by:

Theorem 1.1. For a planar convex compact set $E$, let $2 h=w(E) / \operatorname{cap} E$. Then $0 \leq h=h(E) \leq 1$ and

$$
A(E) \leq \operatorname{cap}^{2} E\left(\pi \beta^{2}+4 h \beta^{\prime} \mathbf{E}\left(\beta^{\prime}, \beta^{\prime-1}\right)\right),
$$

where $\mathbf{E}$ denotes the elliptic integral of the second kind, $\beta^{\prime}=\sqrt{1-\beta^{2}}$, and $\beta=\beta(h)$ is a solution to the equation

$$
h=\beta \mathbf{E}\left(\beta, \beta^{-1}\right)
$$


unique in the interval $0<\beta<1$. In addition, for a fixed cap $E=c$, the right-hand side of (1.1) strictly increases from 0 to $\pi c^{2}$ as $h$ runs from 0 to 1.

Equality occurs in (1.1) if and only if $E$ coincides up to a linear transformation with the set $E^{h}$, symmetric w.r.t. the coordinate axes, complementary to the image $f\left(\mathbb{U}^{*}\right)$ of $\mathbb{U}^{*}=\{z:|z|>1\}$ under a univalent conformal mapping $w=f(z)$ with $f=g \circ \tau$, where

$$
g(\tau)=h+\frac{1}{2} \int_{2}^{\tau} \frac{\tau+\sqrt{\tau^{2}-4 \beta^{2}}}{\sqrt{\tau^{2}-4}} d \tau, \quad \tau=(1 / 2)\left(z+\sqrt{z^{2}-4}\right)
$$

with the principal branches of the radicals.

Figure 1 displays extremal configurations for some typical values of $h$.
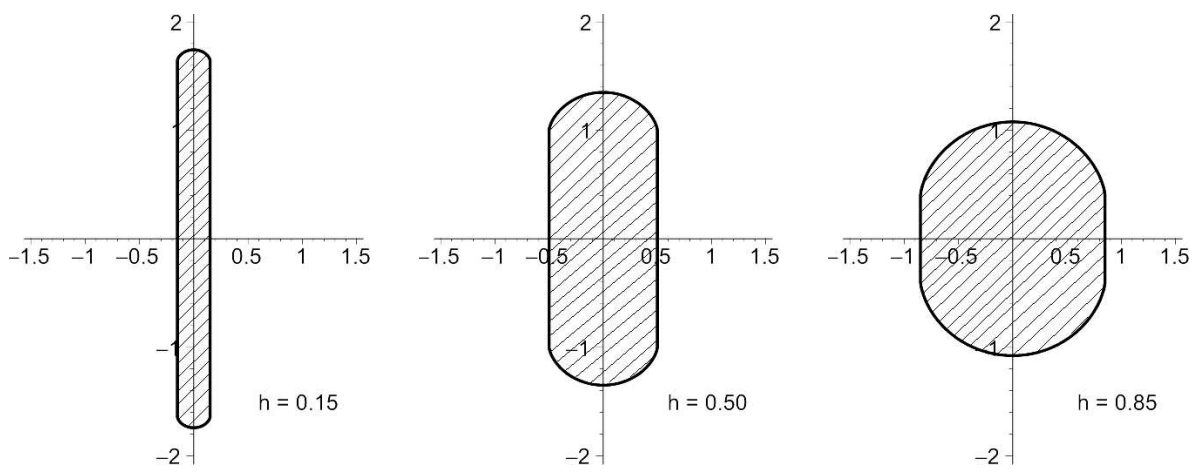

Figure 1. Typical extremal confugurations.

Let $A(h)=\max A(E)$, where the maximum is taken among all convex compact sets $E$ such that $\operatorname{cap} E=1, w(E)=2 h$. Then by Theorem 1.1, $A(h)$ equals the right-hand side of (1.1). The graph of $A(h)$ coincides with a part, for $0 \leq h \leq 1$, of the graph in Figure 2, which shows the maximal area among all compact sets with logarithmic capacity 1 and prescribed projection onto the real axis, as it is explained in Theorem 1.2.

The Proof of Theorem 1.1 given in Section 3 actually leads to a more general result: Inequality (1.1) holds true with the same uniqueness assertion for all compact sets $E$ (connected or not) such that $0 \leq h(E) \leq 1$. However, we prefer to speak about convex sets since the inequalities $0 \leq h(E) \leq 1$ give the whole range of $h(E)$ over the family of all such sets with equalities $h(E)=0$ and $h(E)=1$ only for rectilinear segments and disks, respectively. This follows from the well-known isoperimetric inequalities:

$$
\begin{gathered}
w(E) \leq \frac{1}{\pi} \int_{0}^{\pi} \operatorname{proj}_{0} E d \theta=\frac{1}{\pi} \operatorname{length}(\partial E), \\
\frac{1}{2 \pi} \operatorname{length}(\partial E) \leq\left(\frac{\operatorname{area} E}{\pi}\right)^{1 / 2} \leq \operatorname{cap} E
\end{gathered}
$$




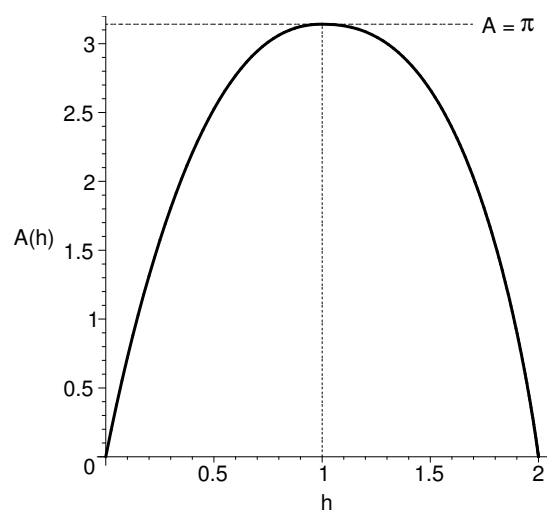

Figure 2. Graph of $A(h)$

cf. [10, pp. 8, 164].

In contrast, the range of $h(E)$ over the set of all continua (= connected compact sets) $E$ is not known. There is an open question, first referenced by Erdös, Herzog and Piranian [5], and later commented on by Ch. Pommerenke $[\mathbf{1 1}]$ to find $\max h(E)$. Erdös et al. conjectured that max $h(E)$ would be 1 ; however, Pommerenke gave an counterexample, $E_{6}$, the symmetric star with six rays, for which $h\left(E_{6}\right)>1$. An easy computation shows that for $E_{3}$, the symmetric star with three rays, that $h\left(E_{3}\right)>h\left(E_{6}\right)$. However, counter to intuition, there are intermediate stars (between $E_{3}$ and $E_{6}$ ) which show that $E_{3}$ cannot be the extremal configuration for max $h(E)$. This remark points out that the problem on the maximal area of $E$ among all continua $E$ with prescribed $h(E)>1$ is potentially quite difficult.

A characteristic of a compact set $E$, dual to the width, is the diameter of $E$ which can be defined as

$$
\operatorname{diam} E=\max _{0 \leq \theta \leq \pi} \operatorname{proj}_{\theta} E .
$$

In $[\mathbf{1}$, Theorem 2], we found the maximal area $A(d)=\max A(E)$ among all continua $E$ such that cap $E=1, \operatorname{diam} E=2 d$. The range of $d=d(E)$, if $E$ is connected and cap $E=1$, is given by the classical inequalities $1 \leq d \leq 2$. The first of them is due to G. Pólya [9] and the second one is due to G. Faber [6]. The upper bound for $d$ shows that the range of the length of projection of $E$ onto a fixed axis, say on $\mathbb{R}$, is

$$
0 \leq \operatorname{proj}_{0} E \leq 4
$$

For a half of this range, when the projection is between 0 and 2 , the arguments used to prove Theorem 1.1 show also that (1.1) holds true with the same uniqueness assertion for all compact sets $E$ such that cap $E=1$ and $0 \leq \operatorname{proj}_{0} E \leq 2$. This result combined with Theorem 2 in [1] gives: 
Theorem 1.2. Let $E$ be a compact set in $\mathbb{C}$ such that cap $E=1$ and $\operatorname{proj}_{0} E=2 h$, where $0 \leq h \leq 2$. Then

$$
A(E) \leq \begin{cases}\pi \beta^{2}+4 h \beta^{\prime} \mathbf{E}\left(\beta^{\prime}, \beta^{\prime-1}\right), & \text { if } \quad 0 \leq h \leq 1, \\ \pi \beta^{2}-2 \pi h(\beta-1), & \text { if } \quad 1 \leq h \leq 2,\end{cases}
$$

where $\beta=\beta(h), 0 \leq \beta \leq 1$ is defined by (1.2) in the first case and $1 \leq \beta \leq 2$ is the unique solution to the equation $h=\beta-(\beta-1) \log (\beta-1)$ in the second case. In addition, the right-hand side of (1.4) strictly increases from 0 to $\pi$ as $h$ runs from 0 to 1 and strictly decreases from $\pi$ to 0 as $h$ runs from 1 to 2 .

For $0 \leq h \leq 1$, extremal configurations are described in Theorem 1.1. For $1 \leq h \leq 2$, equality occurs in (1.4) if and only if $E$ coincides up to a linear transformation with the complement to the image $f\left(\mathbb{U}^{*}\right)$ of $\mathbb{U}^{*}$ under a conformal mapping $f(z)=h+\int_{1}^{z} \varphi(z ; h) d z$, where $\varphi$ maps $\mathbb{U}^{*}$ conformally onto the complement of the "double anchor"

$$
\begin{aligned}
F(\beta, \psi)=[-i \beta, i \beta] & \cup\left\{\beta e^{i t}: \frac{\pi}{2}-\psi \leq t \leq \frac{\pi}{2}+\psi\right\} \\
\cup & \left\{\beta e^{i t}: \frac{3 \pi}{2}-\psi \leq t \leq \frac{3 \pi}{2}+\psi\right\}
\end{aligned}
$$

with $\psi=(1 / 2) \cos ^{-1}\left(8 \beta^{-1}-8 \beta^{-2}-1\right)$.

For the right-hand side of (1.4) we will keep notation $A(h)$, where now $0 \leq h \leq 2$; in context of Theorem $1.1, A(h)$ was defined only for $0 \leq h \leq 1$.

\section{Geometry and closed form of the extremals.}

In Lemma 2.1 we summarize well-known symmetrization results necessary for our main proofs, see $[\mathbf{3}, \mathbf{7}, \mathbf{2}]$ and $[\mathbf{1}]$.

Lemma 2.1. For any compact set $E$, let $E^{* *}$ be the result of successive Steiner symmetrizations of $E$ w.r.t. the real and imaginary axes, respectively. Then

$$
A\left(E^{* *}\right)=A(E), \quad \operatorname{proj}_{0} E^{* *}=\operatorname{proj}_{0} E, \quad \operatorname{cap} E^{* *} \leq \operatorname{cap} E
$$

with the sign of equality in the third relation if and only if $E^{* *}$ coincides with $E$ a.e. up to shifts in the directions of the coordinate axes.

It follows from (2.1) that in proving Theorem 1.2 we may restrict ourselves with continua possessing double Steiner symmetry w.r.t. the coordinate axes. Furthermore, since cap $E, w(E)$, $\operatorname{proj}_{\theta} E$, and $(A(E))^{1 / 2}$ all change linearly w.r.t. scaling, we may assume in what follows that cap $E=1$. Then, $w(E)$ in Theorem 1.1 may vary in between 0 and 2 , and $\operatorname{proj}_{0} E$ in Theorem 1.2 varies in between 0 and 4 . 
If $E$ is connected and Steiner symmetric, then $\Omega_{E}=\overline{\mathbb{C}} \backslash E$ is a simply connected domain containing the point $z=\infty$. Let $f$ be a conformal mapping from $\mathbb{U}^{*}$ onto $\Omega_{E}$. If cap $E=1$, we can normalize $f$ such that

$$
f(\zeta)=\zeta+a_{0}(f)+a_{1}(f) \zeta^{-1}+\cdots .
$$

The set of all analytic functions univalent in $\mathbb{U}^{*}$ and normalized by $(2.2)$ constitute the standard class $\Sigma$, see $[\mathbf{3}, 4]$ and $[\mathbf{8}]$.

For $f \in \Sigma$, let $D_{f}=f\left(\mathbb{U}^{*}\right)$ and $E_{f}=\mathbb{C} \backslash D_{f}$. Our previous considerations show that the problem in Theorem 1.2 is equivalent to the problem on the maximal omitted area for the class $\Sigma$ under the additional constraint

$$
\operatorname{proj}_{0} E_{f}=2 h,
$$

$0 \leq h \leq 2$. The set of functions $f \in \Sigma$ such that $0 \in E_{f}$ and projection of $E_{f}$ onto $\mathbb{R}$ coincides with the segment $[-h, h]$ will be denoted by $\Sigma^{h}$. The omitted area $A_{f}=A\left(E_{f}\right)$ can be computed as

$$
A_{f}=\pi\left(1-\sum_{n=1}^{\infty} n\left|a_{n}(f)\right|^{2}\right) .
$$

Let $A_{\Sigma}(h)=\sup _{f \in \Sigma^{h}} A_{f}$. Since the area functional $A_{f}$ is lower semicontinuous, the existence of an extremal function, at least one for each $h$, easily follows from the compactness of the class $\Sigma^{h}$. Thus, the proof of Lemma 2.2 is standard (see [1] and [2]) and left to the reader.

Lemma 2.2. For every $0 \leq h \leq 2$, there exists $f \in \Sigma^{h}$ such that $A_{f}=$ $A_{\Sigma}(h)$. In addition, $A_{\Sigma}(h)$ is continuous in $0 \leq h \leq 2$.

Let $f$ be an extremal function in $\Sigma^{h}, 0<h<2$. By Lemma 2.1, we may assume that $E_{f}$ possesses Steiner symmetry w.r.t. the coordinate axes. This implies that the boundary $L_{f}=\partial E_{f}$ contains two "free" parts $L_{f r}^{+}=\{z \in$ $\left.\partial E_{f}: \Im z>0,|\Re z|<h\right\}$ and $L_{f r}^{-}=\left\{z: \bar{z} \in L_{f r}^{+}\right\}$. The double symmetry of $E_{f}$ and a standard subordination argument easily imply that $L_{f r}^{+}$is Jordan rectifiable, see similar considerations in [1].

For the "non-free" part of $L_{f}$ there are two possibilities: Either it consists of two vertical segments (possibly degenerate) $I^{ \pm}=\{w= \pm h+i s:|s| \leq$ $\left.s_{f}\right\}, 0 \leq s_{f} \leq 2$, or it consists of two horizontal segments $I_{ \pm}=\{w= \pm t$ : $\left.h_{f} \leq t \leq h\right\}, 0 \leq h_{f} \leq h$.

Let $l_{f r}^{+}=\left\{e^{i \theta}: \theta_{0}<\theta<\pi-\theta_{0}\right\}$ and $l_{f r}^{-}=\left\{e^{i \theta}: e^{-i \theta} \in l_{f r}^{+}\right\}$be the "free arcs", i.e., $l_{f r}^{ \pm}$are the preimages of $L_{f r}^{ \pm}$under the mapping $f$. Similarly, let $l_{n f}^{ \pm}=f^{-1}\left(I^{ \pm}\right)$if the non-free boundary is vertical and $l_{n f}^{ \pm}=f^{-1}\left(I_{ \pm}\right)$if it is horizontal.

Lemma 2.3. For a fixed $h, 0 \leq h \leq 2$, let $f \in \Sigma^{h}$ be extremal for $A_{\Sigma}(h)$ possessing Steiner symmetry w.r.t. the coordinate axes and having a vertical non-free boundary. Then: 
(i) $\left|f^{\prime}(z)\right|=\beta$ with some $0<\beta<1$ for all $z \in l_{\text {fr }}^{ \pm}$;

(ii) $\left|f^{\prime}\left(e^{i \theta}\right)\right|$ strictly decreases from $\rho=\left|f^{\prime}(1)\right|$ to $\beta$ as $\theta$ runs from 0 to $\theta_{0}$. Proof. First, we show that $\left|f^{\prime}(z)\right|$ is constant a.e. on $l_{f r}^{+}$. Since $L_{f r}^{+}$is Jordan rectifiable it follows that the nonzero finite limit

$$
f^{\prime}(\zeta)=\lim _{z \rightarrow \zeta, z \in \overline{\mathbb{U}^{*}}} \frac{f(z)-f(\zeta)}{z-\zeta} \neq 0, \infty
$$

exists a.e. on $l_{f r}$. This easily follows from [12, Theorem 6.8] applied to the univalent function $1 / f(1 / z)$. Assume that

$$
0<\beta_{1}=\left|f^{\prime}\left(e^{i \theta_{1}}\right)\right|<\left|f^{\prime}\left(e^{i \theta_{2}}\right)\right|=\beta_{2}<\infty
$$

for $e^{i \theta_{1}}, e^{i \theta_{2}} \in l_{f r}^{+}$. Note that (2.3) and (2.4) allow us to apply the two point variational formulas of Lemma 5 in [1], see also [2, Lemma 10] for similar variational formulas for analytic functions univalent in the unit disk $\mathbb{U}=$ $\{z:|z|<1\}$. Namely, for fixed positive $k_{1}, k_{2}$ such that $0<k_{1}<1<k_{2}$ and $k_{1} \beta_{1}^{-1}>k_{2} \beta_{2}^{-1}$ and fixed $\varphi>0$ small enough, we consider the two point variation $\widetilde{D}$ of $D_{f}$ centered at $w_{1}=f\left(e^{i \theta_{1}}\right)$ and $w_{2}=f\left(e^{i \theta_{2}}\right)$ with inclinations $\varphi$ and radii $\varepsilon_{1}=k_{1} \varepsilon, \varepsilon_{2}=k_{2} \varepsilon$ respectively, see Section 2 in [1]. Computing the change in the area by formula (2.11) [1], we find

$$
\begin{aligned}
\text { Area }(\mathbb{C} \backslash \widetilde{D})-\text { Area }\left(\mathbb{C} \backslash D_{f}\right) & =\frac{2 \pi \varphi-\sin 2 \pi \varphi}{2 \sin ^{2} \pi \varphi} \varepsilon^{2}\left(k_{2}^{2}-k_{1}^{2}\right)+o\left(\varepsilon^{2}\right) \\
& >0
\end{aligned}
$$

for all $\varepsilon>0$ small enough. Similarly, applying formula (2.10) [1], we get

$$
\log \frac{R(\widetilde{D}, \infty)}{R\left(D_{f}, \infty\right)}=\left[\frac{\varphi(2+\varphi)}{6(1+\varphi)^{2}} \frac{k_{1}^{2}}{\beta_{1}^{2}}-\frac{\varphi(2-\varphi)}{6(1-\varphi)^{2}} \frac{k_{2}^{2}}{\beta_{2}^{2}}\right] \varepsilon^{2}+o\left(\varepsilon^{2}\right)>0
$$

for all $\varepsilon>0$ small enough and $\varphi$ chosen such that the expression in the brackets is positive.

Inequalities (2.5) and (2.6), via a standard subordination argument, lead to a contradiction with the extremality of $f$ for $A_{\Sigma}(h)$. Thus $\left|f^{\prime}\left(e^{i \theta}\right)\right|=\beta$ a.e. on $l_{f r}^{ \pm}$with some $\beta>0$.

Since $E_{f}$ is Steiner symmetric w.r.t. $\mathbb{R}$, the strict monotonicity of $\left|f^{\prime}\left(e^{i \theta}\right)\right|$ in $0 \leq \theta<\theta_{0}$ follows from Lemma $3\left[\mathbf{1}\right.$. To prove that $\left|f^{\prime}\left(e^{i \theta}\right)\right|>\beta$ for all $e^{i \theta} \in l_{n f}^{+}$, we assume that $\beta=\left|f^{\prime}\left(e^{i \theta_{1}}\right)\right|>\left|f^{\prime}\left(e^{i \theta_{2}}\right)\right|=\beta_{2}$ with $e^{i \theta_{1}} \in l_{f r}^{+}$and some $e^{i \theta_{2}} \in l_{n f}^{+}$. Then applying the two point variation as above, we get inequalities (2.5) and (2.6), again, via a subordination argument, contradicting the extremality of $f$ for $A_{\Sigma}(h)$. Hence, $\left|f^{\prime}\left(e^{i \theta}\right)\right| \geq \beta$ for all $e^{i \theta} \in l_{n f}^{ \pm}$, which combined with the strict monotonicity property of $\left|f^{\prime}\right|$ leads to the strict inequality $\left|f^{\prime}\left(e^{i \theta}\right)\right|>\beta$ for $e^{i \theta} \in l_{n f}^{ \pm}$. 
To prove that $\left|f^{\prime}\right|=\beta$ everywhere on $l_{f r}^{+}$, we consider the function $g(z)=$ $1 / f(1 / z)$. The double symmetry property of Lemma 2.1 implies that $D_{g}=$ $g(\mathbb{U})$ is Jordan rectifiable and starlike w.r.t. $w=0$. Therefore, it is a Smirnov domain, see [12, p. 163]. This implies that $\log \left|g^{\prime}(z)\right|=\log \left|f^{\prime}(1 / z)\right|-$ $2 \log |z f(1 / z)|$, and therefore $\log \left|f^{\prime}(1 / z)\right|$, can be represented by the Poisson integral

$$
\log \left|f^{\prime}(1 / z)\right|=\frac{1}{2 \pi} \int_{0}^{2 \pi} P(r, \theta-t) \log \left|f^{\prime}\left(e^{-i t}\right)\right| d t
$$

with boundary values defined a.e. on $\mathbb{T}=\{z:|z|=1\}$, see $[\mathbf{1 2}$, p. 155]. Equation (2.7) along with relations $\left|f^{\prime}\right|=\beta$ a.e. on $l_{f r}^{ \pm}$and $\left|f^{\prime}\right|>\beta$ everywhere on $l_{n f}^{ \pm}$shows that $1=\left|f^{\prime}(\infty)\right| \geq \beta$ with equality only for the function $f(z) \equiv z$.

If $l_{n}^{+}=\emptyset$ or consists of a single point, then the previous arguments show that $\left|f^{\prime}\right|=\beta$ identically on $\mathbb{U}^{*}$. Therefore, $f(z) \equiv z$, which can happen only for $h=1$. Hence, $l_{n}^{+} \neq \emptyset$ and therefore $0<\theta_{0}<\pi / 2$ if $h \neq 1$. Let $v$ be a bounded harmonic function on $\mathbb{U}$ with boundary values $\log (\beta)$ on $l_{f r}^{ \pm}$and $\log \left|f^{\prime}\left(e^{-i \theta}\right)\right|$ on $l_{n f}^{ \pm}$. Then $v(z)-\log \left|f^{\prime}(1 / z)\right|$ has nontangential limit 0 a.e. on $\mathbb{T}$. Therefore, $v(z)-\log \left|f^{\prime}(1 / z)\right| \equiv 0$ on $\overline{\mathbb{U}}$. Hence, $\left|f^{\prime}\right|=\beta$ everywhere on $l_{f r}^{ \pm}$.

To show that $f^{\prime}$ is continuous at $\pm e^{ \pm i \theta_{0}}$, we note that by the reflection principle, $f$ can be continued analytically through $l_{n f}^{+}$and $f^{\prime}$ can be continued analytically through $l_{f r}^{+}$. This implies that $f$ can be considered as a function analytic in a slit disk $\left\{z:\left|z-e^{i \theta_{0}}\right|<\varepsilon\right\} \backslash\left[(1-\varepsilon) e^{i \theta_{0}}, e^{\theta_{0}}\right]$ with $\varepsilon>0$ small enough.

Using the Julia-Wolff lemma, see [12, Proposition 4.13], boundedness of $\log f^{\prime}$, and well-known properties of the angular derivatives, see [12, Propositions $4.7,4.9]$, one can prove that $f^{\prime}$ has a finite limit $f^{\prime}\left(e^{i \theta_{0}}\right),\left|f^{\prime}\left(e^{i \theta_{0}}\right)\right|=\beta$, along any path in $\overline{\mathbb{U}^{*}}$ ending at $e^{i \theta_{0}}$ and by double symmetry at $-e^{ \pm i \theta_{0}}$ and $e^{-i \theta}$. The details of this proof are similar to the arguments in Lemma 13 in $[2]$.

Since $\left|f^{\prime}\right|$ takes its minimal values on $\mathbb{T}$, it follows that $\left|f^{\prime}(z)\right|>\beta$ for all $z \in \mathbb{U}^{*}$. In particular, $\beta<\left|f^{\prime}(\infty)\right|=1$. The proof is complete.

Summing up the results of this section we can prove the following lemma, which allows us to find a closed form for extremal functions.

Lemma 2.4. Let $f \in \Sigma^{h}, 0 \leq h \leq 2$, be extremal for $A_{\Sigma}(h)$ having the vertical non-free boundary. Then $\varphi(z)=z f^{\prime}(z)$ maps $\mathbb{U}^{*}$ univalently onto a domain $\Omega(\beta, \rho)=\overline{\mathbb{C}} \backslash\left\{\overline{\mathbb{U}}_{\beta} \cup[-\rho, \rho]\right\}$ with $\rho=1+\sqrt{1-\beta^{2}}$ and some $\beta=\beta(h) \in(0,1)$. 
Proof. Considering boundary values of $\varphi$, we have $\arg \varphi\left(e^{i \theta}\right)=0$ for $0 \leq$ $\theta \leq \theta_{0}$ since $\Re f\left(e^{i \theta}\right)$ is constant for such $\theta$. Since $\left|\varphi\left(e^{i \theta}\right)\right|=\left|f^{\prime}\left(e^{i \theta}\right)\right|$ strictly increases in $0<\theta<\theta_{0}, \varphi$ maps the arc $\left\{e^{i \theta}: 0 \leq \theta \leq \theta_{0}\right\}$ continuously and one-to-one onto the segment $\{w=t: \beta \leq t \leq \rho\}$ with $\rho=\left|f^{\prime}(1)\right|$.

For $\theta_{0} \leq \theta \leq \pi-\theta_{0},\left|\varphi\left(e^{i \theta}\right)\right|=\beta$. Since $|\varphi(z)|>\beta$ for all $z \in \mathbb{U}^{*}$ it follows that $\varphi^{\prime}\left(e^{i \theta}\right) \neq 0$ for $\theta_{0}<\theta<\pi-\theta_{0}$. Hence $\varphi$ is locally univalent on $l_{f r}^{+}$and therefore $\arg \varphi\left(e^{i \theta}\right)$ strictly increases when $\theta$ runs from $\theta_{0}$ to $\pi-\theta_{0}$.

Let $\vec{n}(\theta)$ be the inner unit normal to $L_{f r}^{+}$at $f\left(e^{i \theta}\right)$. Then $0 \leq \arg \vec{n}(\theta) \leq \pi$ for $\theta_{0} \leq \theta \leq \pi-\theta_{0}$ since $E_{f}$ is Steiner symmetric. Since $\arg \vec{n}(\theta)=\theta+$ $\arg f^{\prime}\left(r^{i \theta}\right)=\arg \varphi\left(e^{i \theta}\right)$, the total variation of $\arg \varphi\left(e^{i \theta}\right)$ on $l_{f r}^{+}$is $<\pi$. This together with the equalities $\arg \varphi\left(e^{i \theta_{0}}\right)=0$ and $\arg \varphi\left(-e^{-i \theta_{0}}\right)=\pi$ shows that $\varphi$ maps $l_{f r}^{+}$continuously and one-to-one onto the upper semicircle $\left\{\beta e^{i \psi}: 0<\psi<\pi\right\}$.

Since $E_{f}$ possesses double symmetry w.r.t. the coordinate axes it follows that $\varphi$ maps $\mathbb{T}$ continuously and one-to-one in the sense of boundary correspondence onto the boundary of $\Omega(\beta, \rho)$. Now by the argument principle, $\varphi$ maps $\mathbb{U}^{*}$ conformally and one-to-one onto $\Omega(\beta, \rho)$. Since $\varphi^{\prime}(\infty)=$ $f^{\prime}(\infty)=1$, an easy computation shows that $\rho=1+\sqrt{1-\beta^{2}}$. The lemma is proved.

\section{Proof of the theorems.}

Proof of Theorem 1.2. By Lemma 2.1, we may restrict ourselves to connected compact sets, which are Steiner symmetric w.r.t. the coordinate axes. Let $E$ be such a continuum extremal for $A_{\Sigma}(h), 0 \leq h \leq 2$ and let $f \in \Sigma^{h}$ map $\mathbb{U}^{*}$ onto $\Omega(E)$.

First we consider the case when the non-free boundary is vertical. By Lemma 2.4, $\varphi=z f^{\prime}$ maps $\mathbb{U}^{*}$ conformally onto $\Omega(\beta, \rho)$ with $\rho=1+$ $\sqrt{1-\beta^{2}}$ and some $0<\beta<1$. The function $\varphi$ can be represented as $\varphi=\beta\left(g^{-1}\left(\beta^{-1} g(z)\right)\right.$, where $g(z)=z+1 / z$ is Joukowski's function. Therefore,

$$
f(z)=h+\beta \int_{1}^{z} z^{-1} g^{-1}\left(\beta^{-1} g(z)\right) d z .
$$

Changing the variable of integration $\tau=g(z)$, we get

$$
f(z)=h+\frac{1}{2} \int_{2}^{\tau} \frac{\tau+\sqrt{\tau^{2}-4 \beta^{2}}}{\sqrt{\tau^{2}-4}} d \tau,
$$

which gives (1.3). Since $\Re f(i)=0$ and $\tau(i)=0$, we find from (3.1),

$$
h=\frac{1}{2} \Re \int_{0}^{2} \frac{\tau+\sqrt{\tau^{2}-4 \beta^{2}}}{\sqrt{\tau^{2}-4}} d \tau=\beta \int_{0}^{\beta} \sqrt{\frac{1-\beta^{-2} x^{2}}{1-x^{2}}} d x,
$$


which is equivalent to (1.2). From (3.2) it is clear that $\beta \mathbf{E}\left(\beta, \beta^{-1}\right)$ strictly increases in $\beta$. Since

$$
\lim _{\beta \rightarrow 0^{+}} \beta \mathbf{E}\left(\beta, \beta^{-1}\right)=0 \quad \text { and }\left.\quad \beta \mathbf{E}\left(\beta, \beta^{-1}\right)\right|_{\beta=1}=1,
$$

it follows that for every fixed $0 \leq h \leq 1$, (1.2) has exactly one solution in $0 \leq \beta \leq 1$. Moreover, this shows that for $1<h \leq 2$, (1.2) has no solutions and therefore extremal continua with the vertical non-free boundary can exist only for $0 \leq h \leq 1$.

The case of extremal continua with horizontal non-free boundary was studied in [1, Theorem 2], which proves (1.4) for $1 \leq h \leq 2$ and shows, in particular, that extremal continua with horizontal non-free boundary can exist only for $1 \leq h \leq 2$. In addition, in case $h=1$ the unit disk $\overline{\mathbb{U}}$ is the unique extremal configuration of the problem under consideration.

In case $1 \leq h \leq 2$, the maximal area $A(h)$ was found in [1, Theorem 2]. To compute $A(h)$ for $0 \leq h \leq 1$, we consider the function $f \in \Sigma^{h}$, such that $E_{f}$ is extremal for the problem under consideration and symmetric w.r.t. the coordinate axes. Applying the standard line integral formula to compute $A(h)=A\left(E_{f}\right)$, we get

$$
\begin{aligned}
A\left(E_{f}\right)=\frac{1}{2} \Im \int_{\partial E_{f}} \bar{w} d w & =\frac{1}{2} \Im \int_{L_{n f}} \bar{w} d w+\frac{1}{2} \Im \int_{L_{f r}} \bar{w} d w \\
& =2 h v_{0}+\frac{1}{2} \Im \int_{L_{f r}} \bar{w} d w,
\end{aligned}
$$

where

$$
v_{0}=\Im f\left(e^{i \theta_{0}}\right)=\frac{1}{2} \Im \int_{2}^{2 \beta} \frac{\tau+\sqrt{\tau^{2}-4 \beta^{2}}}{\sqrt{\tau^{2}-4}} d \tau=\int_{\beta}^{1} \frac{x+\sqrt{x^{2}-\beta^{2}}}{\sqrt{1-x^{2}}} d x .
$$

Now, taking the condition $\left|f^{\prime}(z)\right|=\beta$ for $z \in l_{\text {fr }}$ into account, we find the integral over the free boundary:

$$
\begin{aligned}
\frac{1}{2} \Im \int_{L_{f r}} \bar{w} d w & =\frac{1}{2} \Re \int_{l_{f r}} f\left(e^{i \theta}\right) e^{-i \theta} \overline{f^{\prime}\left(e^{i \theta}\right)} d \theta \\
& =\frac{\beta^{2}}{2} \Re \int_{-\pi}^{\pi} \frac{f\left(e^{i \theta}\right) e^{i \theta}}{e^{2 i \theta} f^{\prime}\left(e^{i \theta}\right)} d \theta-\frac{\beta^{2}}{2} \Re \int_{l_{n f}} \frac{f\left(e^{i \theta}\right)}{e^{i \theta} f^{\prime}\left(e^{i \theta}\right)} d \theta \\
& =\frac{\beta^{2}}{2} \Im \int_{\mathbb{T}} \frac{f(z)}{z^{2} f^{\prime}(z)} d z-2 \beta^{2} h \int_{0}^{\theta_{0}} \frac{d \theta}{\left|f^{\prime}\left(e^{i \theta}\right)\right|} \\
& =\frac{\beta^{2}}{2} \Im \operatorname{Res}\left[\frac{f(z)}{z^{2} f^{\prime}(z)}, \infty\right]-2 \beta^{2} h \int_{0}^{\theta_{0}} \frac{d \theta}{\left|f^{\prime}\left(e^{i \theta}\right)\right|} \\
& =\pi \beta^{2}-2 \beta^{2} h \int_{0}^{\theta_{0}} \frac{d \theta}{\left|f^{\prime}\left(e^{i \theta}\right)\right|} .
\end{aligned}
$$


To find $\int_{0}^{\theta_{0}} \frac{d \theta}{\left|f^{\prime}\left(e^{i \theta}\right)\right|}$, we change the variable of integration $z=(1 / 2)(\tau+$ $\left.\sqrt{\tau^{2}-4}\right)$, then we get

$$
\begin{aligned}
\int_{0}^{\theta_{0}} \frac{d \theta}{\left|f^{\prime}\left(e^{i \theta}\right)\right|} & =2 \int_{2 \beta}^{2} \frac{d \tau}{\sqrt{4-\tau^{2}}\left(\tau+\sqrt{\tau^{2}-4 \beta^{2}}\right)} \\
& =\beta^{-2} \int_{\beta}^{1} \frac{x-\sqrt{x^{2}-\beta^{2}}}{\sqrt{1-x^{2}}} d x .
\end{aligned}
$$

Combining all these computations, we obtain

$$
A(h)=\pi \beta^{2}+4 h \int_{\beta}^{1} \frac{\sqrt{x^{2}-\beta^{2}}}{\sqrt{1-x^{2}}} d x=\pi \beta^{2}+4 h \beta^{\prime} \mathbf{E}\left(\beta^{\prime}, \beta^{\prime-1}\right),
$$

which proves (1.4) for $0 \leq h \leq 1$.

The monotonicity of $A(h)$ for $1 \leq h \leq 2$ was established in [1]. To prove that $A(h)$ is monotone in $0 \leq h \leq 1$, one can show by direct computation that $A^{\prime}(h)>0$ for $0<h<1$. Here we prefer another argument of a general nature. Since cap $E=1$, diam $E \geq 2>2 h$. Since $\partial E^{h}$ is smooth, it follows that for every $h^{\prime}, h<h^{\prime} \leq 1$ there is $\theta^{\prime}=\theta^{\prime}\left(h^{\prime}\right), 0<\theta^{\prime}<\pi$, such that $\operatorname{proj}_{\theta^{\prime}} E^{h}=2 h^{\prime}$. This implies that the continuum $E^{h, \theta^{\prime}}=\left\{z: e^{i \theta^{\prime}} z \in E^{h}\right\}$ is admissible for the problem on $A_{\Sigma}\left(h^{\prime}\right)$ but not extremal since $E^{h, \theta^{\prime}}$ clearly does not have Steiner symmetry w.r.t. $\mathbb{R}$. Therefore $A_{\Sigma}\left(h^{\prime}\right)>A\left(E^{h, \theta^{\prime}}\right)=$ $A_{\Sigma}(h)$. The Proof of Theorem 1.2 is complete.

Proof of Theorem 1.1. Let $E$ be a compact set such that cap $E=1$ and $w(E)=2 h, 0<h<1$ and let $E^{h}$ be the continuum from the Proof of Theorem 1.2 extremal for $A_{\Sigma}(h)$. It follows from Theorem 1.2 that $A(E) \leq$ $A\left(E^{h}\right)$ with the sign of equality only if $E$ coincides a.e. with $E^{h}$ up to a linear transformation. Note that $w\left(E^{h}\right)=2 h$. Indeed, if $w\left(E^{h}\right)=2 h^{\prime}<2 h$, then $A(h)=A\left(E^{h}\right) \leq A\left(h^{\prime}\right)$ contradicting the strict monotonicity property of $A(h)$. This shows that $E^{h}$ has the maximal area among all compact sets, connected or not, with logarithmic capacity 1 and prescribed width $2 h$.

To complete the Proof of Theorem 1.1, we consider the function $f \in \Sigma^{h}$, which maps $U^{*}$ onto $\Omega\left(E^{h}\right)$. By Lemma $2.4, \varphi=z f^{\prime}$ maps $\mathbb{U}^{*}$ onto $\Omega(\beta, \rho)$ with certain $\rho \geq \beta \geq 0$. Since $\mathbb{C} \backslash \Omega(\beta, \rho)$ is starlike w.r.t. the origin, it follows from the classical Alexander's theorem, see [4, p. 43], that $L_{f}$ is convex. Thus, $E^{h}$ is a unique up to a linear transformation convex compact set, which maximizes the area among all such sets with cap $E=1$ and prescribed width $w(E)=2 h$. 


\title{
References
}

[1] R.W. Barnard, K. Pearce and A.Yu. Solynin, An isoperimetric inequality for logarithmic capacity, Annales Academiæ Scientiarum Fennicæ, Mathematica, 27 (2002), 419-436, MR 2003g:30039.

[2] R.W. Barnard and A.Yu. Solynin, Local variations and minimal area problem for Caratheodory functions, submitted.

[3] V.N. Dubinin, Symmetrization in geometric theory of functions of a complex variable, Uspehi Mat. Nauk, 49 (1994), 3-76 (in Russian); English translation in Russian Math. Surveys, 49(1) (1994), 1-79, MR 96b:30054, Zbl 0830.30014.

[4] P.L. Duren, Univalent Functions, Grundlehren Math. Wiss., 259, Springer-Verlag, New York, 1983, MR 85j:30034, Zbl 0514.30001.

[5] P. Erdös, F. Herzog and G. Peranian, Metric properties of polynomials, J. Analyse Math., 6 (1958), 123-148, MR 21 \#123, Zbl 0088.25302.

[6] G. Faber, Neuer Beweis eines Koebe-Bieberbachschen Satzes über konforme Abbildung, Sitzgsber. Math.-Phys. Kl. Bayer. Akad. Wiss. München, 1916, 39-42.

[7] W.K. Hayman, Multivalent Functions, Cambridge Univ. Press, Cambridge, 1958, MR 21 \#7302, Zbl 0082.06102.

[8] J.A. Jenkins, Univalent functions and conformal mappings (2nd ed.), Ergebnisse der Mathematik und ihrer Grenzgebiete, 18, Springer, Berlin, 1958, MR 20 \#3288, Zbl 0083.29606.

[9] G. Pólya, Beitrag zur Verallgemeinerung des Verzerrungssatzes auf maehrfach zusammenhängende Gebiete, S.-B. Preuss. Akad., 1928, 280-282.

[10] G. Pólya and G. Szegö, Isoperimetric Inequalities in Mathematical Physics, Princeton University Press, Princeton, NJ, 1951, MR 13,270d, Zbl 0044.38301.

[11] Ch. Pommerenke, On metric properties of complex polynomials, Michigan Math. J., 8 (1961), 97-115, MR 27 \#1564, Zbl 0100.25503.

[12] _ Boundary Behaviour of Conformal Maps, Grundlehren Math. Wiss., 299, Springer-Verlag, Berlin, 1992, MR 95b:30008, Zbl 0762.30001.

Received May 20, 2002. This paper was written during the third author's visit to Texas Tech University, Fall 2001/Spring 2002. This author thanks the Department of Mathematics and Statistics at Texas Tech for the wonderful atmosphere and working conditions during his stay in Lubbock. The research of the third author was supported in part by Russian Fund for Fundamental Research, grant no. 00-01-00118a.

\section{Department of Mathematics and Statistics}

Texas Tech University

LuBBock, TX 79409

E-mail address: barnard@math.ttu.edu

\author{
Department of Mathematics and Statistics \\ TeXas Tech University \\ LuBBock, TX 79409 \\ E-mail address: pearce@math.ttu.edu
}


UNIVERSITY OF ARKANSAS

Fayetteville, AR 72701

E-mail address: solynin@uark.edu 